\title{
Capacidades de innovación para la gestión del diseño en PYME de empaques plásticos flexibles
}

\author{
Design Management Innovation Capabilities in Flexible Plastics Packaging SMEs
}

\author{
Miguel David Rojas López ${ }^{(1)}$, Felipe Zapata Roldán ${ }^{(2)}$ \\ (1) Doctor en Ingeniería de Sistemas. Profesor Asociado, Facultad de Minas, Universidad Nacional de Colombia. Medellín, Colombia. \\ mdrojas@unal.edu.co \\ (2) Magíster en Ingeniería. Profesor Asociado, Facultad de Diseño Industrial, Universidad Pontificia Bolivariana. Medellín, Colombia. \\ felipe.zapata@upb.edu.co
}

Recibido 15 de mayo de 2014. Modificado 23 de noviembre de 2014. Aprobado 12 de febrero de 2015.

DOI: http://dx.doi.org/10.16924/riua.v0i41.455

\section{Palabras clave \\ Capacidades dinámicas, diseño industrial, estrategia, ge- renciamiento organizacional, gestión de la innovación.}

\section{Resumen}

La gestión del diseño es una aplicación de los métodos de la gerencia de proyectos que lleva menos de una década de estudio, pero muchos autores indican que es clave para el desempeño de los productos de las empresas. En este trabajo se presentan los resultados de un análisis de correlación multivariado aplicado en una zona metropolitana de Colombia en veintiuna pequeñas y medianas empresas (PYME) del sub-sector de empaques plásticos flexibles. Con base en este análisis, se encontraron siete variables que permiten traducir cuantitativamente las capacidades de innovación relacionadas con la gestión del diseño y del desarrollo de productos.

\section{Key words}

Dynamic capabilities, industrial design, innovation management, organizational management, strategy.

\begin{abstract}
Design management is an application of project management methods that has been studied for less than a decade, but which, from literature, appears to be a key factor to product performance especially in manufacturing companies. This work presents the results of an empirical measurement and its multivariate correlation on twenty-one Small and Medium Enterprises (SMEs) of the plastic packaging sector. Based on this analysis, seven factors were found that allow the quantitative translation of a set of innovation capabilities related to design and product development management.
\end{abstract}

\section{INTRODUCCIÓN}

Los sectores productivos de un país y las organizaciones que los integran requieren información para la planificación de estrategias y la toma de decisiones. Existe información estadística en Colombia sobre diversos temas, incluidas la manufactura (sector secundario) y la innovación, recolectadas y analizadas por el Departamento Administrativo Nacional de Estadística (DANE) mediante la Encuesta de Innovación Tecnológica (EDIT) y la Encuesta Anual Manufacturera (EAM). La principal desventaja de ambas tomas de información relacionadas con el desarrollo de productos es que al momento de ser analizados y realizados los informes, los datos originales ya tienen varios años de retraso. Por ejemplo, en el caso de la quinta versión de la EDIT (DANE, 2012), cuyo análisis fue publicado en septiembre de 2012, esta comprendía una ventana de tiempo de datos entre 2009 y 2010 que fueron recolectados en 2011. Esta EDIT era la más actualizada al cierre de la investigación en la que se basa este trabajo. De esta manera, dichas encuestas resultan ser una “instantánea” de cómo era la dinámica de innovación del país dos o tres años antes de aparecer los resultados.

Otro inconveniente práctico de instrumentos como la EDIT y la EAM es el manejo de lenguaje técnico y de alto nivel, derivado de sugerencias estandarizadas como las del Manual de Oslo (OECD, 2005). Dentro de los conceptos de competitividad y productividad organizacional se resalta que, dentro de la generación de valor en las actividades productivas, existen factores estrechamente relacionados con el producto y las actividades realizadas para el acceso de dicho producto al mercado (Baker y Sinkula, 2005; Ortega, 2010). Muchos 
de estos factores relacionados con la generación de innovaciones están interconectados con un conjunto de actividades, procesos y metodologías asociadas a la disciplina del diseño, en particular, las actividades de investigación y el aprendizaje organizacional (Best, 2010; Calantone, Cavusgil y Zhao, 2002; Hobday, 1998).

Por otra parte, los estudios realizados en el tema de capacidades de innovación por fuera de Colombia (Amara, Landry, Halilem, \& Traore, 2010; Branzei \& Vertinsky, 2006; Morrison, Rabellotti, \& Pietrobelli, 2006; Teixeira, 2004; Wang, Lu, \& Chen, 2008; Yam, Guan, Pun \& Tang, 2004) no declaran, en su gran mayoría, que son estrictamente de innovación tecnológica, y Colombia no es una excepción (DANE, 2012; Malaver \& Vargas, 2010; Robledo, Malaver, Vargas, \& Charum, 2009). En general, dejan de lado la problemática asociada al detalle del andamiaje para la generación de conocimiento en los procesos y en la conceptualización, diseño y prototipado de los productos.

Adicionalmente, la literatura específica sobre las capacidades de innovación tecnológica contempla diferentes fuentes de capacidades organizacionales directamente asociadas con la innovación tecnológica que se podrían agrupar en seis categorías o dimensiones (Guan \& Ma, 2003; Yam et al., 2004): capacidad de I+D, capacidad de gestión de recursos, capacidad de aprendizaje organizacional, capacidad de gestión estratégica, capacidad de mercadeo y capacidad organizacional. Según estos autores, la acumulación de estas capacidades no es un fin en sí mismo, sino un medio para lograr ciertos objetivos empresariales. El logro de estos objetivos corresponde a lo que se denomina "desempeño empresarial" y puede ser medido por medio de un desempeño innovador entendido como el logro de innovaciones de producto y proceso, y como un desempeño del negocio relacionado con el impacto de la innovación en las ventas, las utilidades, la participación en el mercado y otros elementos cuantificables.

La industria de los empaques flexibles en Colombia, como subsector del gran sector empaques, envases y embalajes (EEE), se configura como un renglón dinámico y transversal de la economía que provee soluciones a otras industrias y, se sostiene sobre las necesidades de envases, empaques y embalajes del sector secundario de la economía (transformación y manufactura). Entre 2003 y 2008, la fracción de la importación de materias primas (polímeros) que se consumieron en el sector empaques y envases en el país osciló entre el 52\% y el 54\% correspondiente a 397.217 toneladas métricas de material, las cuales ascendieron a 502.146 toneladas métricas en 2010 , aumentando en un 33.5\% con respecto al año inmediatamente anterior.

Según reporta Acoplásticos, en 2010 en este sector se tuvo un consumo aparente de 909.000 toneladas métricas de resinas plásticas y de ellas el 54\% en peso promedio fueron para empaques y envases entre el 2008 y el 2010 (Acoplásticos, 2011). Todo esto puesto en el contexto de los factores clave identificados por el estudio desarrollado por el Centro de Pensamiento Estratégico y Prospectivo de la Universidad Externado de Colombia y apoyado por Acoplásticos y el ICIPC (Mahecha, 2011), hacen el sector de EEE interesante para estudiar las dinámicas de los procesos de generación de producto. Los tres factores principales en el trabajo de Mahecha (2011) fueron: innovación, creación de valor agregado y sostenibilidad; $y$, los tres se conjugan en las actividades de diseño de producto.

Este trabajo presenta una propuesta de indicadores de innovación relacionados con la gestión de los proyectos de diseño en pequeñas y medianas empresas del subsector de EEE de la ciudad de Medellín y municipalidades vecinas (Área Metropolitana del Valle de Aburrá).

\section{Aspectos metodológicos}

Se realizó una búsqueda sistemática de medibles en un grupo de trabajos clave que abordaban la problemática del desarrollo de producto, el relacionamiento externo o la generación de bienes de alto valor agregado (DANE, 2010; Guan \& Ma, 2003; Guzmán-Cuevas \& Martínez-Román, 2008; Merrilees, Rundle-Thiele \& Lye, 2011; Robledo et al., 2009). Con base en estos, se generó una relación de cuáles elementos, variables o medibles tenían estos trabajos en común a manera de inspección visual y con taxonomías temporales quedando una lista de 109 elementos, que agrupados temáticamente y por afinidad, se redujeron a 70 variables medibles (resultados no mostrados). Como proceso intermedio se evaluó cuáles podrían ser similares o redundantes, y así el conjunto se redujo a 52 variables/medibles relacionadas con las capacidades de innovación.

El primer filtro de este conjunto de 52 variables, se realizó escogiendo solo aquellas que estuvieran referenciadas en mínimo dos trabajos del conjunto de aquellos referenciados al inicio de esta sección (que incluye la EDIT III del 2010, para la cual el formulario estaba disponible al momento de la ejecución de la investigación). Esto condujo a un conjunto final de 32 medibles y con base en ese subconjunto se ensambló un cuestionario para evaluación por parte de expertos que por medio de calificación independiente ayudaron a reducir el conjunto a 23 medibles.

\section{ENCUESTA ESTRUCTURADA}

Las 23 variables que quedaron después del proceso con los expertos fueron agrupadas por afinidad en seis indicadores (que posteriormente se convirtieron en siete al dividir el último en dos), siguiendo como guía parcial algunas clasificaciones utilizadas en otros trabajos y realizando una asignación de variables a tres dimensiones a manera de taxonomía de construcción propia (Tabla 1). El orden de la numeración de los códigos de las variables coincide con el orden de las preguntas realizadas en la consulta a expertos, el cual cambió al reordenarlas taxonómicamente. En la dimensión organizacional se alojan aquellas variables 


\begin{tabular}{|c|c|c|c|}
\hline DIMENSIÓN & INDICADOR & Cód. & VARIABLE \\
\hline \multirow{7}{*}{ ORGANIZACIONAL } & \multirow{7}{*}{ INTRÍNSECO } & P1 & Vinculación de personal especializado para actividades de innovación \\
\hline & & P2 & Esquema de reconocimiento, incentivos y promoción del recurso humano \\
\hline & & P3 & $\begin{array}{l}\text { Flexibilidad y autonomía en la estructura de la organización que permita que } \\
\text { surjan innovaciones y emprendimientos }\end{array}$ \\
\hline & & P4 & Adaptación a los cambios de mercado y aceptación del riesgo \\
\hline & & P5 & Formulación de una política de desarrollo de producto en la organización \\
\hline & & P6 & $\begin{array}{l}\text { Incorporación de la tecnología (equipos y conocimiento) en las estrategias } \\
\text { de la organización }\end{array}$ \\
\hline & & P19 & $\begin{array}{l}\text { Conciencia estratégica de la posición competitiva de la organización y de los } \\
\text { factores externos a los que está expuesta }\end{array}$ \\
\hline \multirow{9}{*}{$\begin{array}{l}\text { GESTIÓN DEL } \\
\text { CONOCIMIENTO }\end{array}$} & GESTIÓN & P8 & $\begin{array}{l}\text { Gestión integral de los proyectos de generación de nuevos productos } \\
\text { (planificación, evaluación, seguimiento y control) }\end{array}$ \\
\hline & \multirow{3}{*}{ GENERACIÓN } & P7 & Desarrollo de producto en el último año \\
\hline & & $\mathrm{P} 10$ & $\begin{array}{l}\text { Incorporación de metodologías o resultados del Diseño Industrial o del } \\
\text { Diseño Gráfico (u otros Diseños) en los productos de la organización }\end{array}$ \\
\hline & & P11 & $\begin{array}{l}\text { Prueba de los productos antes de salir al mercado (por medio de prototipos, } \\
\text { lotes piloto y otros métodos) }\end{array}$ \\
\hline & \multirow{3}{*}{ APRENDIZAJE } & P9 & $\begin{array}{l}\text { Monitoreo sistematizado del conocimiento interno y externo a la } \\
\text { organización }\end{array}$ \\
\hline & & P12 & $\begin{array}{l}\text { Capacitación de personal a todo nivel (directivo y operativo) que permita la } \\
\text { absorción y asimilación de conocimiento externo a la organización }\end{array}$ \\
\hline & & $\mathrm{P} 13$ & Comprensión de la segmentación del mercado de la organización \\
\hline & \multirow[t]{2}{*}{ TRANSFERENCIA } & P14 & $\begin{array}{l}\text { Adquisición y negociación de tecnología (equipos, insumos, conocimiento, } \\
\text { asesoría técnica) que permite manufactura y desarrollo de nuevos } \\
\text { productos }\end{array}$ \\
\hline & & P22 & $\begin{array}{l}\text { Incorporación del conocimiento en sentido estricto en I+D y conocimiento } \\
\text { de mecanismos de protección a la propiedad intelectual }\end{array}$ \\
\hline \multirow{7}{*}{ RELACIONAMIENTO } & \multirow{7}{*}{ INTERNO } & P15 & $\begin{array}{l}\text { Comunicación permanente entre las áreas involucradas en el desarrollo de } \\
\text { nuevos productos }\end{array}$ \\
\hline & & P16 & $\begin{array}{l}\text { Capacidad de producción y manufactura, habilitada por el conocimiento } \\
\text { generado en el proceso de desarrollo de nuevos productos }\end{array}$ \\
\hline & & P17 & $\begin{array}{l}\text { Utilización de mecanismos gubernamentales de financiación al desarrollo de } \\
\text { innovaciones }\end{array}$ \\
\hline & & P18 & $\begin{array}{l}\text { Articulación con fuentes externas de financiación privada al desarrollo de } \\
\text { nuevos productos }\end{array}$ \\
\hline & & P20 & $\begin{array}{l}\text { Colaboración directa o indirecta con la competencia como aliados, fuentes } \\
\text { de información o generadores de ideas }\end{array}$ \\
\hline & & P21 & $\begin{array}{l}\text { Relacionamiento con actores generadores de ideas y conocimiento } \\
\text { (Universidades, expertos, consultores, centros de investigación, entre otros) }\end{array}$ \\
\hline & & P23 & Frecuencia de uso de ideas de los clientes en los productos \\
\hline
\end{tabular}

Tabla 1. Estructura de dimensiones, indicadores y variables resultado de la consulta a expertos.

relacionadas con factores estratégicos, mentalidad, recursos humanos o de cultura organizacional. La dimensión de gestión del conocimiento recoge todos los factores asociados al flujo, apropiación y uso del conocimiento interno a la empresa, proveniente de actores externos y que se saca al entorno (empresarial, institucional, social y económico). La dimensión de relacionamiento da cuenta de los intercambios de recursos e información que ocurren al interior de la organización entre los agentes participantes de los procesos de desarrollo de nuevos productos; de igual manera, esta dimensión comprende los intercambios desde y hacia la organización, en particular, los relacionados con conocimiento puro y empaquetamientos tecnológicos.

El paso siguiente para la construcción del instrumento fue la formulación de preguntas basadas en cada una de las variables priorizadas, en las que la medida o la forma de medición variaba dependiendo de los autores, ya que en algunos casos eran variables cualitativas puestas en escala Likert para 
poder ser cuantificadas; en otros casos, simplemente eran variables categóricas. Las preguntas se formularon en términos del estado actual o de la percepción del estado de esa variable, y el objetivo del estudio era conocer cuáles eran las relaciones existentes entre las variables identificadas sobre las cuales se construyó el instrumento, por lo cual se optó por volver cuantitativas la mayoría de los medibles en la fase de tabulación de los datos, ya que la mayoría de las respuestas eran variables categóricas (Likert o binarias). En la escala Likert para las preguntas que la requirieron, se dieron opciones en escalas ascendentes de cinco elementos que indagaron por un nivel (de "Nulo" a "Muy alto") o por una frecuencia (de "Nunca” a "Siempre”). Por último, los datos numéricos fueron ingresados y procesados en el software de análisis estadístico Statgraphics ${ }^{\circledR}$ de StatPoint Technologies.

\section{AnÁlisis estadístico}

Dado el bajo número de las PYME manufactureras del subsector específico de EEE encontradas en Medellín y su área metropolitana con base en el directorio de Acoplásticos
(Acoplásticos, 2011) y para determinar si se podían alcanzar los mínimos muestrales en el estudio empírico, se realizó una estimación de la población de PYME en la región de análisis. Se debe tener en cuenta que la Confederación de Cámaras de Comercio de Colombia reportó 3’396.343 establecimientos a nivel nacional en 2011-2012 (Confecámaras, 2012), de los cuales el 19,2\% estaban ubicados en Antioquia y, aproximadamente 652.000 establecimientos estaban ubicados en Medellín y su área metropolitana. Según la Asociación Nacional de Instituciones Financieras (ANIF), cerca del 90\% de estos establecimientos eran PYME (ANIF, 2013).

La ecuación para la determinación de intervalos de confianza y estimación de errores para distribuciones normales (Bartlett, Kotrlik, \& Higgins, 2001) se usó sobre la población de 34.000 PYME, y se calculó que los valores para el tamaño de la muestra de la encuesta $(n=21)$ fueron del 95\% con un error estimado del $13.2 \%$, lo cual es aceptable teniendo en cuenta las distorsiones de las muestras pequeñas y los alcances del estudio.

Con el fin de tener estadísticas descriptivas de las variables, se calcularon las medias y se estimaron los errores. Se hizo

\begin{tabular}{|c|c|c|c|c|}
\hline Variable & Media & Error Estimado & Tendencia de la respuesta & Tema \\
\hline P1 & 0,571 & 0,111 & & \\
\hline P2 & $0,286^{*}$ & 0,101 & No & Existencia de incentivos a nuevas ideas \\
\hline P3 & 3,190 & 0,255 & Medio-alto & \\
\hline P4 & $3,619^{*}$ & 0,161 & Alto & Nivel de adaptación a los cambios en el mercado \\
\hline P5 & $0,714^{*}$ & 0,101 & Sí & Políticas de desarrollo de productos \\
\hline P6 & 3,524 & 0,255 & Casi siempre & \\
\hline P7 & 0,905 & 0,066 & Sí & \\
\hline P8 & $0,381^{*}$ & 0,109 & Sí & Existencia de metodologías de proyectos \\
\hline P9 & 3,143 & 0,295 & Algunas veces & \\
\hline P10 & $3,714^{*}$ & 0,171 & Casi siempre & Frecuencia de los cambios en diseño \\
\hline P11 & 3,762 & 0,266 & Casi siempre & \\
\hline P12 & 3,762 & 0,238 & Casi siempre & \\
\hline P13 & 3,429 & 0,202 & Medio-Alto & \\
\hline P14 & 3,238 & 0,217 & Medio-Alto & \\
\hline P15 & 3,667 & 0,187 & Alto & \\
\hline P16 & 3,333 & 0,174 & Medio-Alto & \\
\hline P17 & 0,476 & 0,112 & & \\
\hline P18 & $0,809 *$ & 0,088 & Sí & Articulación con fuentes de financiación privadas \\
\hline P19 & 3,809 & 0,203 & Alto & \\
\hline $\mathrm{P} 20$ & 3,048 & 0,223 & Medio-Alto & \\
\hline P21 & 2,524 & 0,235 & & \\
\hline P22 & 3,333 & 0,270 & Medio-Alto & \\
\hline P23 & $4,190^{*}$ & 0,177 & Siempre & Opiniones de clientes \\
\hline
\end{tabular}

Tabla 2. Estadísticas descriptivas para las respuestas a la encuesta. Los valores marcados con asterisco $\left({ }^{*}\right)$ se consideran tendencias de medias notables con porcentaje de error bajo. 
un corte a aquellas variables que presentaron un error mayor al $20 \%$ y cuya tendencia se podía establecer con claridad según la conversión de variables categóricas Likert en variables cuantitativas normalizadas. Los menores errores estimados con tendencias claras se obtuvieron en las variables P2, P5, P8 y P18 (incentivos, políticas de I+D claras, existencia de metodologías de gestión de proyectos, y conocimiento de financiación privada respectivamente). Esto quiere decir que entre estas variables existe consistencia en la forma en la que respondieron las PYME de EEE a la encuesta.

\section{ESTUDIO DE CORRELACIÓN Y RESULTADOS}

Se construyó una matriz (no mostrada) de veintitrés por veintitrés (23x23) elementos con base en el coeficiente de correlación de Pearson (Wilcox, 2009), también llamado coeficiente producto-momento de Pearson, calculado entre todas las respuestas a las variables. Para identificar las variables relevantes se usó la "alerta” de correlación significativa que arrojó el software de forma automática y luego se observó la tendencia de cada una de las columnas y filas, siguiendo una metodología modificada del análisis de matriz de impacto cruzado (Godet, 1995), haciendo promedio de los valores absolutos de las correlaciones y escogiendo las de mayores valores promedio. De esta manera, se identificaron aquellas variables que influencian de manera fuerte la dinámica de las otras. En el ejemplo de la Tabla 2, aunque la variable P14 (Negociación de tecnología) también tiene marcas de correlación significativa, la variable P15 (Frecuencia de la comunicación entre las áreas involucradas en desarrollo de producto) en toda la extensión de las variables exhibió mejor tendencia, por lo cual se considera una variable influenciadora. Es decir, su valor positivo cercano a uno (y mayor de 0.5) en todas las celdas de la columna P15 de la Tabla 2, indica que en aquellas empresas encuestadas en las que esta variable era de valores numéricos más altos, otras variables también obtenían valores numéricos altos, por tanto respuestas similares altas o bajas (coincidentes) entre la variable P15 y las demás. Un caso contrario en este ejemplo es la variable P12 (Frecuencia de capacitación del personal) que presentó pocas correlaciones significativas, y donde la tendencia era heterogénea como se puede observar parcialmente en la Tabla 3 .

\begin{tabular}{|c|c|c|c|c|}
\hline & P12 & P13 & P14 & P15 \\
\hline P3 & 0.1552 & 0.1983 & $0.6478^{*}$ & $0.7672^{*}$ \\
\hline P4 & 0.1917 & 0.2502 & $0.4009^{*}$ & $0.5786^{*}$ \\
\hline P5 & 0.2546 & 0.0667 & $0.3721^{*}$ & $0.6307^{*}$ \\
\hline P6 & $0.3777^{*}$ & 0.2909 & $0.7052^{*}$ & $0.7838^{*}$ \\
\hline
\end{tabular}

Tabla 3. Segmento notable de la matriz de correlación entre ocho de las variables que hicieron parte del instrumento de medición. Los valores marcados con asterisco fueron reportados automáticamente como de correlación significativa.
Se verificó que las variables influenciadoras identificadas en la matriz de correlación, según los promedios y tendencias de correlación, dieran cuenta de todos los indicadores propuestos. Se organizaron según la división de dimensiones e indicadores descrita en la Tabla 1, y sólo se dejaron aquellas variables en las que su nivel de influencia estuviera determinado a su vez por la correlación estadística. Esto permitió darle un cuerpo de medibles explicativos a los indicadores, reduciendo el conjunto de variables a siete (Tabla 4).

Según los resultados obtenidos, estos siete medibles y sus actividades o recursos asociados son clave en términos de capacidades para el desarrollo de productos y para la gestión del diseño. Por lo anterior, las PYME de EEE deben poner especial atención a estos factores, bien sea para gestionar adecuadamente los recursos y habilidades que ya poseen o compensar cualquier falencia relacionada.

\begin{tabular}{|c|c|c|}
\hline DIMENSIÓN & INDICADOR & VARIABLE \\
\hline A) ORGANIZACIONAL & 1) INTRÍNSECO & $\begin{array}{l}\text { P2: Esquema de } \\
\text { reconocimiento, } \\
\text { incentivos y promoción } \\
\text { del recurso humano }\end{array}$ \\
\hline \multirow{4}{*}{$\begin{array}{l}\text { B) GESTIÓN DEL } \\
\text { CONOCIMIENTO }\end{array}$} & 2) GESTIÓN & $\begin{array}{l}\text { P8: Gestión integral } \\
\text { de proyectos de } \\
\text { generación de nuevos } \\
\text { productos (planificación, } \\
\text { evaluación, seguimiento } \\
\text { y control) }\end{array}$ \\
\hline & 3) GENERACIÓN & $\begin{array}{l}\text { P10: Incorporación } \\
\text { de metodologías o } \\
\text { resultados del Diseño } \\
\text { Industrial o del Diseño } \\
\text { Gráfico (u otros Diseños) } \\
\text { en los productos de la } \\
\text { organización }\end{array}$ \\
\hline & 4) APRENDIZAJE & $\begin{array}{l}\text { P11: Realización de } \\
\text { pruebas de producto }\end{array}$ \\
\hline & 5) TRANSFERENCIA & $\begin{array}{l}\text { P22: Conocimiento } \\
\text { de mecanismos de } \\
\text { protección a la propiedad } \\
\text { industrial }\end{array}$ \\
\hline \multirow[b]{2}{*}{ C) RELACIONAMIENTO } & 6) INTERNO & $\begin{array}{l}\text { P15: Comunicación } \\
\text { permanente entre las } \\
\text { áreas involucradas en } \\
\text { el desarrollo de nuevos } \\
\text { productos }\end{array}$ \\
\hline & 7) EXTERNO & $\begin{array}{l}\text { P21: Relacionamiento } \\
\text { con actores generadores } \\
\text { de ideas y conocimiento } \\
\text { (Universidades, expertos, } \\
\text { consultores, centros } \\
\text { de investigación, entre } \\
\text { otros) }\end{array}$ \\
\hline
\end{tabular}

Tabla 4. Reformulación de las variables de alimentación a los indicadores y las dimensiones de la propuesta de medición, según resultados del estudio. 
Si se asume que las PYME no tienen desarrolladas las capacidades de creación de nuevos productos, hay validez en esta afirmación al encontrar que los coeficientes de correlación de la variable P10 (Frecuencia de cambios en el diseño de producto) en la columna correspondiente son negativos; es decir, otras capacidades se desarrollan más al disminuir el énfasis en el diseño de producto. Esto va en contra de las premisas de la gestión del diseño que, como condición mínima, requieren que dichos cambios sean constantes y apreciables.

Al relacionar la gestión del diseño con procesos organizacionales, se busca que la gestión de proyectos sea sinérgica con las metodologías de diseño que se insertan a los procesos organizacionales en las PYME. La presencia de estas aparenta ser evidente, pero la variable P8 (uso de metodologías de gestión de proyectos) está correlacionada con las dos variables correspondientes a la explicitación de lineamientos de desarrollo de productos y a la posibilidad de reasignación de personal para desarrollo de nuevos productos (P3 y P6), por lo que a menos de que haya elementos estratégicos claros, no existe un insumo para organizar los procesos de gestión de proyectos, y mucho menos los de gestión del diseño. En este caso en los ambientes donde hay mayor planificación, existen unas mejores condiciones para la adaptación y la respuesta rápida a los mercados, y en otras palabras, las metodologías de diseño y de gestión del diseño, así sea de manera informal, hacen parte de los procesos de la organización.

En general, existe un conjunto de variables relacionadas con capacidades dinámicas, que tienen que ver con la gestión del diseño y se evidencian en las PYME. Algunas de estas capacidades dinámicas identificadas (aprendizaje, producción y estrategia) tienen sus representantes en cada una de las secciones de la encuesta. Se extrae de los resultados cuáles son entradas al sistema, ya que afectan a otras variables (ej. P11, P15, P22 y P23), y cuáles son salidas del sistema y pueden ser asociadas a los resultados del proceso porque se dejan influenciar más en estos sistemas empresariales de las PYME (ej. P2, P3, P4 y P6); es decir, los incentivos a nuevas ideas, la reasignación de personal ante cambios en el mercado, la capacidad de respuesta y la explicitación de lineamientos de desarrollo de productos. Esto último muestra que entre más hábil sea una empresa para desarrollar productos, catalizará sus procesos con incentivos y tendrá más flexibilidad al momento de operar.

\section{Conclusión}

Por medio del estudio de correlación y la indagación instrumental del estado de un conjunto de variables clave, se logró determinar que existen siete variables que son altamente influenciadoras y afectan directamente otros factores asociados a las PYME de EEE. Se encontró que en este tipo de empresas trabajan por proyectos y tienden a incorporar desarrollos de la disciplina del diseño que, por lo general, son probados antes de llegar al mercado.
El EEE es un subsector en el que el conocimiento de los mecanismos de protección a la propiedad intelectual y el relacionamiento con agentes generadores de conocimiento e ideas promueve la generación de innovaciones, y mientras más se fortalezcan estos dos elementos mejores probabilidades de éxito en el mercado tendrán sus productos. Igualmente, es un sector muy competido pero con altas posibilidades de crecimiento e introducción de novedades, por lo que la circulación de conocimiento internamente entre los miembros de las PYME y la estimulación por medio de incentivos a las nuevas ideas, puede generar efectos positivos en la frecuencia y calidad de las nuevas propuestas al mercado. La determinación y características de la influencia de los esquemas de protección a la propiedad industrial en Colombia y en las decisiones estratégicas en la gestión de un proyecto de diseño quedan planteadas para ser desarrolladas en otra investigación.

Es interesante el hallazgo de que la lectura del contexto y el conocimiento del mercado parecen no ser tan relevantes en las PYME de EEE; pesa más el conocimiento de lo que está haciendo un competidor a través de registros de propiedad industrial o por canales informales. Lo anterior, enlazado con la importancia que tienen las pruebas de producto en sus procesos, parecieran indicar que las PYME de este subsector tienden a ofrecer mejoras a las propuestas de los competidores, bien sea en funcionalidad o en costos de producción, pero no en novedad en cuanto a diseño, lo cual queda como una hipótesis abierta.

\section{REFERENCIAS}

Acoplásticos. (2011). Plásticos en Colombia 2011-2012 (XLI., p. 220). Bogotá: Acoplásticos.

Amara, N., Landry, R., Halilem, N. \& Traore, N. (2010). Patterns of Innovation Capabilities in KIBS Firms: Evidence from the 2003 Statistics Canada Innovation Survey on Services. Industry \& Innovation, 17(2), 163-192.

ANIF. (2013). La gran encuesta PYME. Bogotá: ANIF

Baker, W. E. \& Sinkula, J. M. (2005). Market Orientation and the New Product Paradox. Journal of Product Innovation Management, 22(6), 483-502. doi:10.1111/j.15405885.2005.00145.x

Bartlett, J. E., Kotrlik, J. W. \& Higgins, C. C. (2001). Organizational Research : Determining Appropriate Sample Size in Survey Research. Information Technology, Learning, and Performance Journal, 19(1), 43-50.

Best, K. (2010). Fundamentos Del Management Del Diseño. Badalona: Parramon.

Branzei, O. \& Vertinsky, I. (2006). Strategic pathways to product innovation capabilities in SMEs. Journal of Business Venturing, 21(1), 75-105. 
Buijs, J. (2003). Modelling Product Innovation Processes, from Linear Logic to Circular Chaos. Creativity and Innovation Management, 12(2), 76-93. doi:10.1111/14678691.00271

Calantone, R. J., Cavusgil, S. T. \& Zhao, Y. (2002). Learning orientation, firm innovation capability, and firm performance. Industrial Marketing Management, 31(6), 515524. doi:10.1016/S0019-8501(01)00203-6

Confecámaras. (2012). Informe de Gestión (En el marco de la 49a Asamblea de Confecámaras). Bogotá: Confecámaras.

Cooper, R. G. (1996). Overhauling the new product process. Industrial Marketing Management, 25(6), 465-482. doi:10.1016/S0019-8501(96)00062-4

DANE. (2010, June). Encuesta de desarrollo e innovación tecnológica - EDIT III (2005-2006). Bogotá: Departamento Administrativo Nacional de Estadística,

DANE. (2012, September). Encuesta de desarrollo e innovación tecnológica - EDIT V (2009-2010). Bogotá: Departamento Administrativo Nacional de Estadística..

Godet, M. (1995). De la anticipacion a la acción: Manual de prospectiva y estrategia. México: Alfaomega Grupo Editor.

Guan, J., \& Ma, N. (2003). Innovative capability and export performance of Chinese firms. Technovation, 23(9), 737747. doi:10.1016/S0166-4972(02)00013-5

Guzmán-Cuevas, J., \& Martínez-Román, J. (2008). Tipología de la innovación y perfiles empresariales: Una aplicación empírica. Economía Industrial, Trimestre (368), 20.

Hobday, M. (1998). Product complexity, innovation and industrial organisation. Research Policy, 26(6), 689-710. doi:10.1016/S0048-7333(97)00044-9

Landau, R., \& Nathan Rosenberg, E. (1986). The Positive Sum Strategy: Harnessing Technology for Economic Growth. The National Academies Press.

Loo, R. (2002). The Delphi method: a powerful tool for strategic management. Policing: An International Journal of Police Strategies \& Management, 25(4), 762-769. doi:10.1108/13639510210450677

Mahecha, C. A. (2011). Estudio prospectivo de los empaques plásticos flexibles y semirrígidos en Colombia. Bogotá.

Malaver, F., \& Vargas, M. (2010). Capacidades tecnológicas, innovación y competitividad de la industria de Bogotá y Cundinamarca: Resultados de una encuesta de innovación. Bogotá: Fondo Cultural Javeriano de Artes Gráficas.

Merrilees, B., Rundle-Thiele, S., \& Lye, A. (2011). Marketing capabilities : antecedents and implications for B2B SME performance. Industrial marketing management: the international journal for industrial and high-tech firms. - New York, NY [u.a.] : Elsevier, 40.2011, 3, p. 368-375.

Morrison, A., Rabellotti, R., \& Pietrobelli, C. (2006). Global Value Chains and Technological Capabilities: A Framework to Study Industrial Innovation in Developing Countries. (p. application/pdf). KITeS, Centre for Knowledge, Internationalization and Technology Studies, Universita' Bocconi, Milano, Italy. Retrieved from http://www.econbiz.de/en/search/detailed-view/doc/all/ global-value-chains-and-technological-capabilities-aframework-to-study-industrial-innovation-in-developing-countries-morrison-andrea/10005403564/?no_cache $=1$

OECD. (2005). Guidelines for Collecting and Interpreting Innovation Data. OECD Publishing (Organisation for Economic Co-operation and Development).

Ortega, M. J. R. (2010). Competitive strategies and firm performance: Technological capabilities' moderating roles. Journal of Business Research, 63(12), 1273-1281. doi:10.1016/j.jbusres.2009.09.007

Porter, M. E. (1998). Competitive Advantage: Creating and Sustaining Superior Performance (1st ed). Free Press. Retrieved from http://www.amazon.com/dp/0684841460

Robledo, J., Malaver, F., Vargas, M., \& Charum, J. (2009). Encuestas, datos y descubrimiento de conocimiento sobre la innovación en Colombia. Universidad Nacional de Colombia. Retrieved from http://books.google.com.co/ books?id=tIXqZwEACAAJ

Roozenburg, N. F. M., \& Eekels, J. (1995). Product Design: Fundamentals and Methods (p. 422). John Wiley \& Sons.

Sun, Q., Williams, A., \& Evans, M. (2011). A Theoretical Design Management Framework. The Design Journal, 14(1), 112-132. doi:10.2752/175630610X12877385838885

Teixeira, A. A. C. (2004). How Has the Portuguese Innovation Capability Evolved? Estimating a Time Series of the Stock of Technological Knowledge, 1960-2001. Retrieved from http://ideas.repec.org/p/por/fepwps/153.html

Wang, C., Lu, I., \& Chen, C. (2008). Evaluating firm technological innovation capability under uncertainty. Technovation, 28(6), 349-363. doi:10.1016/j.technovation.2007.10.007

Wilcox, R. R. (2009). Basic Statistics: Understanding Conventional Methods and Modern Insights (1st ed.). USA: Oxford University Press.,

Yam, R. C. M., Guan, J. C., Pun, K. F., \& Tang, E. P. Y. (2004). An audit of technological innovation capabilities in chinese firms: some empirical findings in Beijing, China. Research Policy, 33(8), 1123-1140. 


\title{
Heurística de enfoque bidireccional para la solución de problemas de equilibrado de líneas de ensamble
}

\author{
Heuristic of Bidirectional Approach to Solve Assembly Line Balancing Problems
}

\author{
Jorge Michael Burgos Meneses ${ }^{(1)}$ \\ (1) Ingeniero Industrial, Universidad de Córdoba, Montería-Córdoba, Colombia. ing.jmburgos.09@gmail.com \\ Recibido 15 de agosto de 2014. Modificado 12 de diciembre de 2014. Aprobado 14 de enero de 2015.
}

DOI: http://dx.doi.org/10.16924/riua.v0i41.667

\section{Palabras clave}

Algoritmos, equilibrado, heurística, líneas de ensamble, tiempo de ciclo.

\section{Resumen}

En este artículo se describe y evalúa un algoritmo de enfoque bidireccional para resolver los SALBPs-1, el cual se fundamenta en la heurística JOMI, construyendo simultáneamente soluciones desde ambos lados de la matriz de precedencias. Aquí se muestra una evaluación comparativa entre este procedimiento y otros 14 algoritmos similares, utilizando 269 instancias encontradas en la literatura, donde los resultados evidenciaron que el algoritmo propuesto genera muy buenas soluciones al arrojar el mejor promedio de eficiencia con un 91,42\%. De este modo, se presenta otra forma sencilla y factible de resolver los problemas de líneas de ensamble.

\section{Key words}

Algorithm, assembly line, balance, heuristic, cycle time.

\begin{abstract}
This article describes and evaluates a bidirectional approach algorithm to solve SALBPs-1. Such a procedure is based on heuristic JOMI, by building solutions from both sides of the precedence matrix simultaneously. A comparative evaluation among this procedure and 14 other similar algorithms is shown. Two hundred and sixty-nine instances found in the literature were used, the results illustrate that the proposed algorithm produces very good solutions, in this case, throwing the best average efficiency of $91.42 \%$. Thus, another simple and practical way to solve assembly lines problems is presented.
\end{abstract}

\section{INTRODUCCIÓN}

Las líneas de ensamble son componentes esenciales para numerosos procesos industriales de producción, principalmente los asociados a la fabricación de productos similares, en masa y baja variedad (celulares, computadores, televisores, carros, etc). Estas líneas se distinguen por estar formadas por estaciones de trabajo, a través de las cuales circula el producto en proceso hasta ser terminado. Cada una de estas estaciones cuenta con un tiempo idéntico para realizar las operaciones, tareas o actividades asignadas, llamado "tiempo de ciclo".

Por otro lado, el "equilibrado de líneas de ensamble" consiste en la adecuada asignación de tareas a estaciones de trabajo, de tal forma que se respeten las relaciones de precedencia que pueden presentar algunas tareas entre sí, y el tiempo de capacidad de las estaciones de trabajo. Este problema de asignación de tareas es conocido como problema de balanceo de líneas de ensamble (Assembly Line
Balancy Problem, ALBPs) y ha sido clasificado en dos tipos principales, los problemas simples de balanceo de líneas de ensamble (Simple Assembly Line Balancy Problem, SALBP) y los problemas generales de balanceo de líneas de ensamble (General Assembly Line Balancy Problem, GALBP); esta clasificación se detalla en (Baybars, 1986), donde se estudia el desarrollo de los SALBP a través del tiempo.

Los SALBP están limitados sólo por restricciones de precedencia y capacidad (tiempo); sus instancias se pueden representar de la siguiente manera: un grupo de actividades $i(i=1, \ldots, n)$, las cuales son indivisibles, poseen una duración de procesamiento conocida $t i$ y algunas presentan relaciones de precedencia entre sí; se distribuyen en un grupo de estaciones de trabajo $S_{j}(j=1, \ldots, m)$, las cuales presentan un tiempo de duración idéntico (tiempo de ciclo “ $C$ ”), con el fin de maximizar la eficiencia de la línea de ensamble, ya sea minimizando el número de estaciones de trabajo, el 